\title{
AS CONTRIBUIÇÕES DAS SIMULAÇÕES FORCES AND MOTION: basics (html5) e projectile motion (html5), da plataforma phet, para o ensino da mecânica Newtoniana
}

\author{
Artur Araújo Cavalcantel \\ Gilvandenys Leite Sales²
}

\section{RESUMO}

Este trabalho tem o objetivo de avaliar a contribuição das "simulações Forces and Motion: Basics (HTML5) e Projectile Motion (HTML5)", da plataforma PhET Interactive Simulations, para o ensino de alguns conceitos da Mecânica Newtoniana. Para avaliar a contribuição dessas simulações no processo de ensino-aprendizagem, selecionamos 44 alunos de duas turmas de $1^{\circ}$ ano do Ensino Médio de uma escola particular de Fortaleza-CE. A aplicação das simulações foi feita apenas em uma das turmas (Turma Experimental); na outra turma (Turma Controle), foram aplicadas apenas aulas expositivas tradicionais (ou clássicas), nas quais o professor utilizou somente os recursos da lousa e do pincel. Para a análise dos conhecimentos prévios e dos conhecimentos obtidos durante as aulas, foi utilizado o Force Concept Inventory (FCl) - teste que avalia as concepções dos estudantes com relação aos conceitos físicos relacionados à Mecânica. Os resultados da pesquisa indicaram que o desempenho dos alunos da Turma Experimental foi superior ao desempenho dos estudantes da Turma Controle, sinalizando para o fato de que as simulações podem contribuir para o aprendizado dos conceitos de Mecânica Newtoniana.

Palavras-chave: Simulações. Ensino. Física. Mecânica. Força.

THE CONTRIBUTIONS OF THE FORCES AND MOTION SIMULATIONS: basics (html5) and projectile motion (htm/5), of the phet platform, for the teaching of the

\section{newtonian mechanics}

\begin{abstract}
This work aims to evaluate the contribution of the simulations "Forces and Motion: Basics (HTML5) and Projectile Motion (HTML5)", from the platform PhET Interactive

\footnotetext{
1 Possui graduação em Licenciatura em Física pelo Instituto Federal de Educação, Ciência e Tecnologia (IFCE). Atualmente, é professor de Física do Colégio Paiva Andrade e Mestrando em Ensino de Física no Programa de Pós-Graduação em Ensino de Ciências e Matemática (PPGECM) do IFCE. Orcid iD: https://orcid.org/0000-0002-3483-8739. E-mail: arturecoba@hotmail.com

2 Doutorado. Instituto Federal de Educação, Ciência e Tecnologia do Ceará/Fortaleza/Ceará/Brasil. Orcid iD: https://orcid.org/0000-0002-6060-2535. Lattes: http://lattes.cnpq.br/9075418972296296
} 
Simulations, to the teaching of some concepts of Newtonian mechanics. To evaluate the contribution of theses simulations on the teaching-learning process, we selected 44 students from two groups of first year of high school from a private school of Fortaleza-CE. The application of the simulations was made in only one of the two groups (Experimental Group); in the other one (Control Group), only traditional expositive classes (or classical classes) were applied, in which the teacher used only the resources of blackboard and marker. To the analysis of previous knowledge and knowledge obtained after the classes, the Force Concept Inventory (FCI) - a test that evaluates the students' conceptions about the physical concepts related to Mechanics - was used. The results of research indicate that the performance of the students of the Experimental Class was superior to the performance of the students of the Control Class, signaling to the fact that the simulations can contribute to the learning of the concepts of Newtonian Mechanics.

Keywords: Simulations. Teaching. Physics. Mechanic. Force.

\section{LAS CONTRIBUCIONES DE LAS SIMULACIONES FORCES AND MOTION: basicS}

(html5) y projectile motion (html5), de la plataforma phet, para la enseñanza de la mecánica newtoniana

\section{RESUMEN}

Este trabajo tiene el objetivo de evaluar la contribución de las simulaciones "Forces and Motion: Basics (HTML5) y Projectil Motion (HTML5)", de la plataforma PhET Interactive Simulations, para la enseñanza de conceptos de la Mecánica Newtoniana. Para evaluar la contribución de estas simulaciones en el proceso de enseñanza-aprendizaje, seleccionamos a 44 alumnos de dos clases de $1^{\circ}$ año de la Enseñanza Media de una escuela particular de Fortaleza-CE. La aplicación de las simulaciones fue hecha solamente en una de las clases (Grupo Experimental); en la otra clase (Grupo Control), se aplicaron apenas clases expositivas tradicionales (o clásicas), en las que el profesor utilizó solamente los recursos de la pizarra y del pincel. Para el análisis de los conocimientos previos y de los conocimientos posteriores a las clases, se utilizó el Force Concept Inventory (FCl) - test que evalúa las concepciones de los estudiantes con relación a los conceptos físicos relacionados a la Mecánica. Los resultados de la investigación indican que el desempeño de los alumnos de lo Grupo Experimental fue superior al desempeño de los estudiantes de lo Grupo Control, señalando para el hecho de que las simulaciones pueden contribuir al aprendizaje de los conceptos de Mecánica Newtoniana.

Palabras clave: Simulaciones. Enseñanza. Física. Mecánica. Fuerza.

\section{INTRODUÇÃO}

É de conhecimento geral que a Física é uma das disciplinas no Ensino Básico responsável por grande parte das reprovações. Esse fato é muitas vezes relacionado às dificuldades na compreensão de alguns fenômenos 
físicos por parte dos alunos, o que, por consequência, os leva a terem certo desinteresse ou mesmo a odiar estudar a disciplina. Além disso, os adolescentes possuem uma dificuldade em absorver conhecimentos que sejam abstratos e que envolvam cálculos matemáticos (PAPALIA; OLDS; FELDMAN, 2013).

Um problema na aprendizagem de Física pode estar relacionado aos métodos de ensino utilizados por grande parte dos professores que, muitas vezes, recorrem apenas às aulas expositivas tradicionais e a um conjunto de exercícios para fazer com que os alunos fixem o conhecimento.

Um dos artifícios que os professores podem utilizar, visando solucionar essa deficiência na aprendizagem e, por conseguinte, aumentar a motivação e o engajamento dos alunos em sala de aula são as Tecnologias Digitais de Informação e Comunicação (TDIC). O uso de tais recursos digitais pode, portanto, tornar o processo de ensino-aprendizagem mais eficaz. As TDIC estão presentes no cotidiano dos jovens contemporâneos - ou "nativos digitais", conforme nomeados por Prensky (2001) -, que cresceram inseridos nessa cultura digital. Contudo, não são utilizadas exclusivamente por esse público, participando também da vida das pessoas que nasceram em gerações anteriores e que, hoje, tendem a interagir com essas tecnologias (PRENSKY, 2001).

Nesse quadro, os avanços dessas tecnologias digitais nos mostram que há diferentes possibilidades de metodologias de ensino em que o aluno pode interagir com objetos digitais de aprendizagem como, por exemplo, os laboratórios virtuais e as simulações.

Nesta pesquisa, temos como objetivo avaliar a contribuição de duas simulações computacionais da plataforma PhET Interactive Simulations da Universidade do Colorado Boulder - Forces and Motion: Basics (HTML5) e Projectile Motion (HTML5) -, para o processo de ensino e aprendizagem da mecânica newtoniana.

Cabe ressaltar, ainda, que a pesquisa foi realizada com base em dados gerados a partir de observações das aulas de mecânica newtoniana, 
que duraram dois meses, em duas turmas de $1^{\circ}$ ano do Ensino Médio de um colégio particular de Fortaleza - CE.

Em relação à organização, o artigo encontra-se dividido em cinco seções, além desta primeira - reservada à apresentação das considerações iniciais - e da sétima - destinada à exposição das considerações finais. $\mathrm{Na}$ segunda seção, abordaremos a pertinência da aplicação de simulações digitais para o ensino de Física; na terceira, discutiremos, de forma mais específica, acerca das duas simulações PhET utilizadas por nós: Forces and Motion: Basics (HTML5) e Projectile Motion (HTML5); na quarta, faremos observações concernentes ao teste Force Concept Inventory (FCI); na quinta, apresentaremos os procedimentos metodológicos do estudo e, finalmente, na sexta seção, faremos a análise dos dados da pesquisa.

\section{AS SIMULAÇÕES APLICADAS AO ENSINO DE FÍSICA}

É notável como a tecnologia vem crescendo, nos últimos anos, e mudando os hábitos da sociedade em geral. Isso pode ser percebido no ambiente de sala de aula, no qual o aluno contemporâneo, como mencionamos, pode ser visto como um nativo digital, sendo conhecedor de vários ambientes virtuais, tendo habilidades para compreender uma linguagem iconográfica e, consequentemente, atentando para textos predominantemente visuais, em detrimento dos verbais (SALES, 2017).

Nesse sentido, o uso de simulações virtuais pode ser uma estratégia eficiente para engajar os alunos nas atividades propostas em sala de aula e tornar o conhecimento teórico mais acessível para eles. Além disso, convém lembrar que a Física "lida com materiais que, muitas vezes, estão fora do alcance dos sentidos do ser humano tais como partículas subatômicas" (MEDEIROS; MEDEIROS, 2002, p. 77) e que o ensino da disciplina em questão se caracteriza pela presença de vários conceitos que são abstratos para os alunos, como a ausência de atrito ou resistência do ar. Em vista disso, é pertinente apontar que as simulações virtuais podem facilitar o trabalho do professor, na medida em que a representação animada dos fenômenos físicos possibilita que o aluno os compreenda melhor. 
As simulações computacionais vão muito além do que uma simples animação. Elas englobam várias classes de tecnologias, que vão do vídeo à realidade virtual e podem ser categorizadas quanto ao grau de interatividade que proporcionam entre $O$ aprendiz e 0 computador (MEDEIROS; MEDEIROS, 2002). Elas também permitem que o professor otimize seu tempo em aulas predominantemente teóricas ou até mesmo em aulas experimentais, na medida que não há um tempo gasto com montagem ou com a familiarização dos alunos com os equipamentos. Sobre isso, Studart (2015, p. 6) sintetiza que a "fácil manipulação das variáveis experimentais permite formular e testar hipóteses em pouco tempo".

Ainda no que diz respeito ao grau de interatividade entre o aprendiz e o computador, as simulações digitais são modelos computacionais que representam situações ou fenômenos reais ou hipotéticos que permitem ao usuário explorar as consequências de manipular ou modificar os parâmetros do modelo (CLARK et al., 2009). Esta é uma das propostas de um dos projetos mais conhecidos de simulações de ciências no mundo: o Physics Educational Technology, doravante PhET.

Fundado em 2002 por Carl Wieman, vencedor do Prêmio Nobel de Física, o projeto PhET, da Universidade de Colorado Boulder, cria simulações interativas gratuitas de ciências e de matemática. As simulações PhET baseiam-se numa extensa pesquisa em educação e envolvem os alunos através de um ambiente intuitivo, em que eles aprendem através da exploração e da descoberta (WIEMAN; PERKINS; ADAMS, 2008).

O principal objetivo das simulações PhET é proporcionar aprendizagem ativa por meio de exploração, dando a possibilidade ao aluno construir seus próprios significados e entendimentos. Sobre a participação direta dos alunos com as simulações PhET, Wieman, Adams e Perkins (2009, p. 683) afirmam que:

Descobrimos que os alunos não conseguem entender a ciência na simulação apenas observando. Eles devem interagir ativamente com a simulação. A maior parte do aprendizado ocorre quando a estudante se faz perguntas que guiam sua exploração da simulação e sua descoberta das respostas. Quando os alunos se envolvem em uma exploração tão autônoma, aprendem melhor. Por exemplo, 
estudantes não-científicos sem conhecimento prévio de física são capazes de fornecer explicações muito boas de uma onda eletromagnética depois de menos de uma hora interagindo com a simulação de "Ondas de rádio" (WIEMAN; ADAMS; PERKINS, 2009, p. 683, tradução nossa³).

Além disso, todas as simulações são testadas com um grande número de alunos e alteradas conforme os resultados dos feedbacks das próprias simulações (WIEMAN; PERKINS; ADAMS, 2008). Levando em conta as observações de Wieman, Perkins e Adams (2008), podemos assinalar que:

- A maioria dos estudantes não consegue distinguir, de imediato, o que são detalhes relevantes e irrelevantes dentro da simulação. Por isso, é importante que o professor faça com que o aluno explore a simulação e descubra as suas funcionalidades aos poucos, respeitando seu próprio tempo.

- Uma simulação não é tão eficiente na aprendizagem do aluno quando o professor apenas demonstra situações hipotéticas, privando o aluno de manuseá-la ou modificá-la.

- Em algumas situações, o uso das simulações pode facilitar o entendimento de um experimento real. Isso se dá porque a simulação pode fornecer ao aluno a percepção de algo microscópico no experimento real.

- Simulações permitem focar no modelo que queremos explorar, eliminando elementos não essenciais que inevitavelmente atrapalham a interpretação de experimentos reais. É o caso, por exemplo, do lançamento de projéteis, situação em que a resistência do ar sempre estará presente em um experimento real, o que pode atrapalhar 0

\footnotetext{
3 No original: "We find that students are not able to make sense of the science in the simulation just from watching. They must interact actively with the simulation. Most of the learning occurs when the student is asking herself questions that guide her exploration of the simulation and her discovery of the answers. When students engage in such self-driven exploration, they learn better. For example, nonscience students with no prior knowledge of physics are able to provide quite good explanations of an electromagnetic wave after less than an hour playing with the 'Radio Waves' simulation" (WIEMAN; PERKINS, ADAMS, 2008, p. 683).
} 
entendimento do aluno na compreensão dos conceitos e na realização dos cálculos.

Ademais, torna-se pertinente fazer menção a outros estudos que enfatizam a eficiência de simulações computacionais, como é o caso de Finkelstein et al. (2005), que acreditam que alunos que realizam experimentos de Física com simulações computacionais compreendem conceitos tão bem ou melhor do que alunos que usaram equipamento físico. Este resultado gera esperanças aos professores de Física de países como o Brasil, na medida que a maioria dos laboratórios nacionais não fornecem um aparato apropriado de aulas experimentais nas mais diversas áreas da Física.

Rutten et al. (2012), por sua vez, após terem feito uma ampla revisão de literatura dos trabalhos que tocam esse tema, descrevem algumas das vantagens da utilização de simulações computacionais visando promover uma aprendizagem mais efetiva. Entre outros aspectos, os autores destacam a possibilidade de o aluno explorar minuciosamente situações hipotéticas de um experimento, interagindo com a sua versão simplificada ou completa, podendo, assim, mudar a escala temporal dos eventos. Além disso, os alunos podem realizar tarefas e resolver problemas de modo mais rápido, em um ambiente que simula a realidade. Com sua pesquisa, os autores concluíram que há evidências concretas de que o uso de simulações computacionais pode melhorar o ensino tradicional.

Outros estudos (BRANSFORD; BROWN; COOKING, 2000; MIRANDA, 2013; PODOLEFSKY, 2010; WIEMAN, 2014) também fornecem importantes descobertas sobre a utilização de simulações na sala de aula e comparam as vantagens e desvantagens do ensino tradicional com e sem o auxílio das simulações computacionais.

Como já foi mencionado, nesse trabalho, utilizaremos algumas simulações do projeto PhET para trabalhar os conceitos de mecânica. Um dos motivos de escolhermos este projeto está relacionado ao fato de que ele permite que os alunos acessem as simulações fácil e livremente e que eles possam manipulá-las, dentre outros dispositivos, em seus próprios aparelhos celulares, na medida que o professor os orienta, ao projetar a 
simulação na lousa. Além disso, o projeto é multilíngue e oferece, em sua plataforma, sugestões de atividades (ARANTES; MIRANDA; STUDART, 2010; PERKINS et al., 2006, WIEMAN et al., 2010) que o professor pode utilizar como atividade para casa, por exemplo.

Feitos esses apontamentos de caráter geral sobre o uso de simulações no ensino de Física, torna-se necessário descrever, de modo mais específico, as simulações do PhET que foram utilizadas com os alunos: Forces and Motion: Basics (HTML5) e Projectile Motion (HTML5). Essa é a tarefa em que nos deteremos na próxima seção.

\section{AS SIMULAÇÕES PhET UTILIZADAS: forces and motion: basics (html5) e projectile motion (html5)}

Nesse trabalho, utilizamos duas simulações do software PhET que, como foi dito anteriormente, é um projeto fundado em 2002 por Carl Wieman e desenvolvido pela Universidade do Colorado Boulder. Essa plataforma foi escolhida por, além das razões já relatadas, apresentar simulações interativas gratuitas de Física (além de Biologia, Química, Ciências da Terra e Matemática). Além disso, essas simulações são bem dinâmicas e de fácil entendimento, possuem uma aparência lúdica e apresentam uma linguagem clara. Esse conjunto de características torna as simulações PhET adequadas para serem trabalhadas com alunos do Ensino Médio (MAKUCH; MARTINS, 2018).

A seguir, são apresentadas as simulações trabalhadas exclusivamente com os alunos da Turma Experimental, no intervalo dos dois meses (14 aulas) em que a pesquisa foi realizada.

\section{Forces and Motion: Basics (HTML5)}

O Forces and Motion: Basics (HTML5) contém simulações relacionadas ao movimento de alguns objetos, como, por exemplo, uma geladeira e uma caixa. Nas simulações, o usuário pode trabalhar situações de movimento, alterando os valores numéricos da força aplicada, da força de atrito e da massa que será movimentada. 
É possível verificar, por exemplo, como se comportam os valores numéricos da velocidade e da aceleração em situações hipotéticas criadas pelo próprio usuário e, assim, explorar conceitos da mecânica newtoniana relacionados às Leis de Newton e ao movimento de corpos.

$\mathrm{Na}$ Figura 1, apresentada abaixo, sintetizamos essa discussão, ilustrando o funcionamento do Forces and Motion: Basics (HTML5):

FIGURA 1 - Imagem ilustrativa do Forces and Motion: Basics (HTML5)

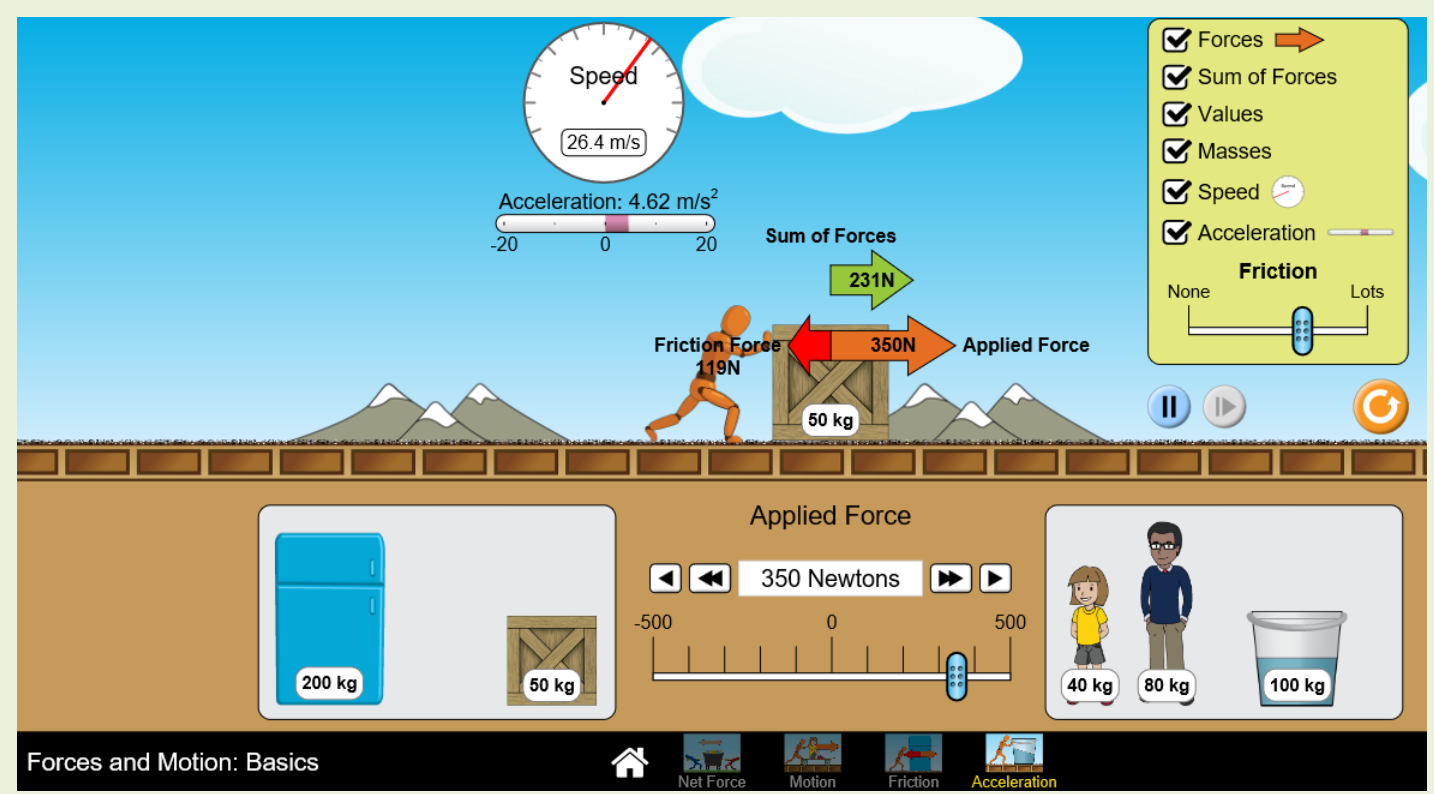

Fonte: PhET Interactive Simulations ${ }^{4}$.

\section{Projectile Motion (HTML5)}

O Projectile Motion (HTML5) apresenta simulações de lançamento de projéteis. Nelas, o usuário pode trabalhar situações de movimento, alterando os valores numéricos da velocidade, do ângulo e da altura de lançamento, da massa e do diâmetro do projétil, além da gravidade local e da resistência do ar. Assim, é possível observar o que acontece com o movimento de diferentes corpos em lançamentos simulados hipoteticamente pelo próprio usuário, permitindo que ele explore conceitos da mecânica newtoniana relacionados ao movimento de corpos.

4 Disponível em: <https://phet.colorado.edu/pt_BR/simulation/forces-and-motion-basics>. Acesso em: 21 de outubro de 2018. 
Na Figura 2, exibida abaixo, resumimos essa discussão, ilustrando o funcionamento do Projectile Motion (HTML5):

FIGURA 2 - Imagem ilustrativa do Projectile Motion (HTML5)

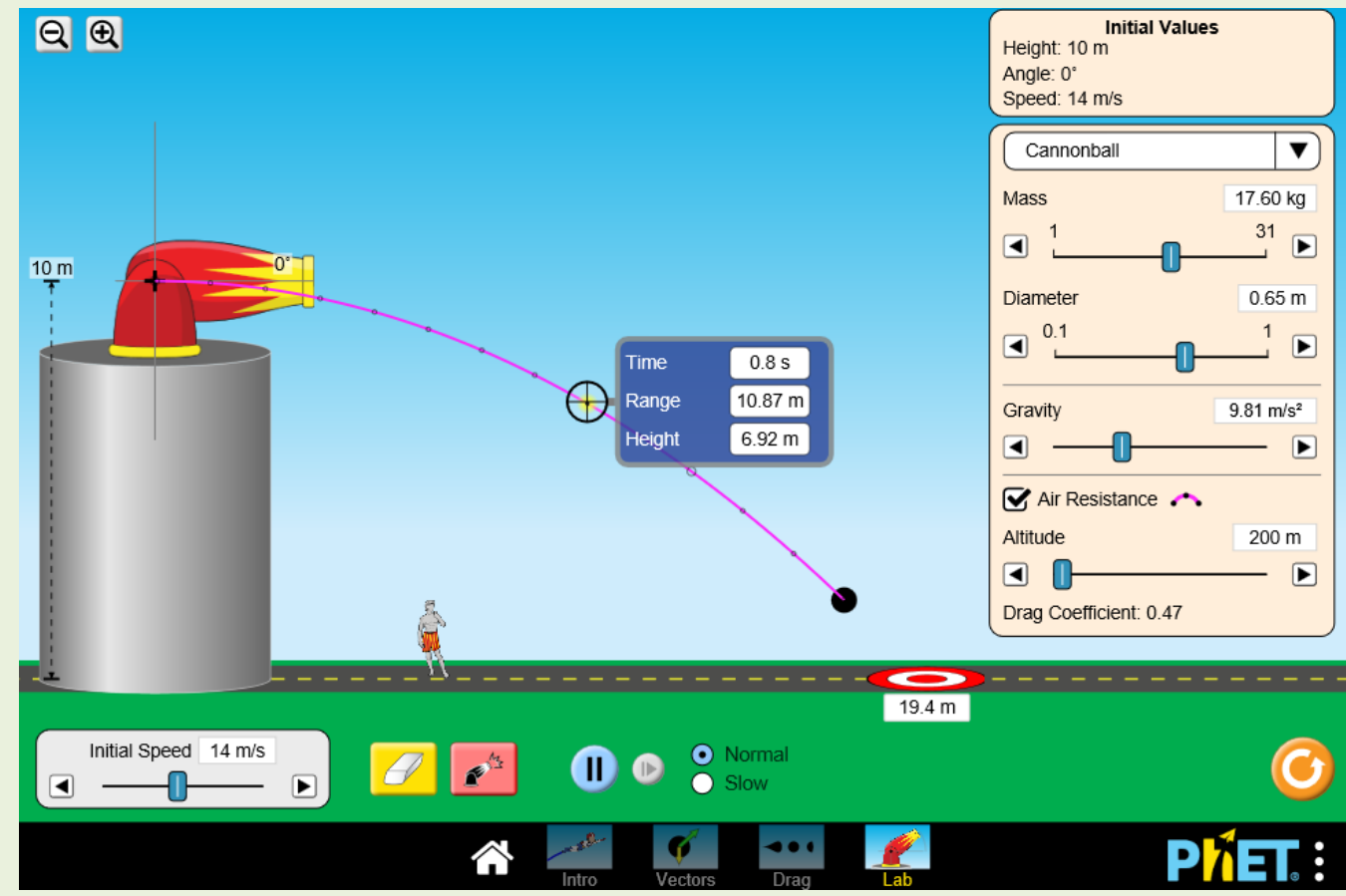

Fonte: PhET Interactive Simulations 5 .

Feitas essas observações mais específicas sobre o Forces and Motion: Basics (HTML5) e sobre o Projectile Motion (HTML5), abordaremos, na próxima seção, o pré-teste e o pós-teste que utilizamos na pesquisa: o Force Concept Inventory (FCI).

\section{O FORCE CONCEPT INVENTORY (FCI)}

O Force Concept Inventory (doravante FCl) - ou Inventário dos Conceitos de Força (ICF), em português - é um teste desenvolvido por Hestenes, Wells e Swackhammer, em 1992, projetado para avaliar a compreensão dos estudantes dos conceitos básicos da mecânica newtoniana (HESTENES; WELLS; SWACKHAMMER, 1992).

5 Disponível em: <https://phet.colorado.edu/pt_BR/simulation/projectile-motion>. Acesso em: 21 de outubro de 2018. 
O referido teste pode ser usado para diversas finalidades, mas geralmente é utilizado para avaliar a eficácia de uma metodologia ou de uma proposta de ensino, comparando aulas tradicionais com aulas interativas, nas quais os alunos participam de forma mais ativa no processo de ensino-aprendizagem (HESTESNES; HALLOUN, 1995).

Atualmente, o $\mathrm{FCl}$ é um teste amplamente utilizado para acompanhar o grau de assimilação dos conceitos de mecânica de estudantes em todos os níveis de ensino e para avaliar, comparativamente, a eficácia de diferentes métodos de ensino da mecânica newtoniana (HOPKINSON; NAKAHARA; ABDULLA, 2018; CHARLES et al., 2015; ARTAMONOVA; MOSQUERA-MOSQUERA; MOSQUERA-ARTAMÓNOV, 2017; CABALLERO ef al., 2012).

O teste aborda conceitos das seguintes áreas da mecânica: Primeira, Segunda e Terceira Lei de Newton, tipos de força, superposição de forças e Cinemática. É estruturado com 30 questões de múltipla escolha, com alternativas de A à E. Cada questão contém apenas uma alternativa que está relacionada aos conceitos da mecânica newtoniana (a alternativa correta); as outras quatro são referentes a distratores ou a conceitos intuitivos previamente estabelecidos na pesquisa de Halloun e Hestenes (1985).

A seguir, na Figura 3, apresentamos, a título de exemplificação, a questão 19 do $\mathrm{FCl}$, traduzida por nós, para mostrar um dos conceitos newtonianos abordados no teste, assim como os distratores apresentados nela: 
FIGURA 3 - Questão 19 do FCl, traduzida

A imagem abaixo mostra as posições de dois blocos, que se deslocam para a direita da imagem. Cada posição difere da anterior em um tempo de 0,2 segundo.

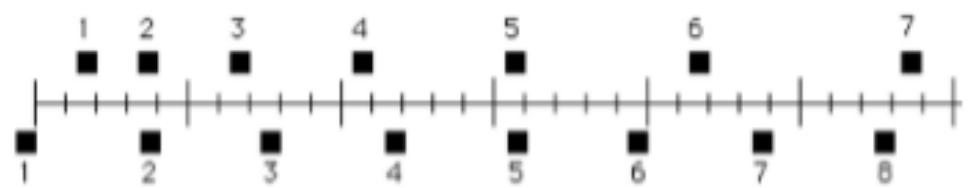

A partir das informações, podemos dizer que os blocos:

a) não possuem a mesma velocidade em nenhum instante.

b) possuem a mesma velocidade somente no instante 2 .

c) possuem a mesma velocidade somente no instante 5 .

d) possuem a mesma velocidade somente nos instantes 2 e 5 .

e) possuem a mesma velocidade em algum momento durante o intervalo de tempo entre os instantes 3 e 4 .

Fonte: Elaborado pelos autores, com base no material disponível no $\mathrm{FCl}^{6}$.

A questão acima trabalha com os conceitos de Movimento Retilíneo Uniforme (MRU) e Movimento Retilínio Uniformemente Variado (MRUV), que dizem respeito à Cinemática ${ }^{7}$. A resposta correta dela corresponde à alternativa "e". As alternativas "b", "c" e "d" são distratores ligados ao conceito intuitivo "mesma velocidade por consequência da mesma posição"; ou seja, marcá-la implica que o aluno não consegue distinguir velocidade de posição. Já a alternativa "a" representa um distrator relacionado ao conceito intuitivo "maior velocidade por consequência de uma maior aceleração"; ou seja, para o aluno que assinala essa alternativa, a aceleração é equivalente à velocidade.

Há também outros conceitos intuitivos não newtonianos ao longo do inventário8, embora estes não sejam contemplados pela questão

6 O acesso ao conteúdo do $\mathrm{FCl}$ é restrito para professores. Caso o professor queira acessar as questões do $\mathrm{FCl}$, precisará fazer $\mathrm{O}$ cadastro na página: https://www.physport.org/assessments/.

7 "É a parte da Mecânica que trata do repouso e do movimento apenas descrevendo-os, isto é, sem preocupar-se com as causas que determinam o estado de repouso ou as características do estado de movimento" (HELOU; GUALTER; NEWTON, 2007, p 16).

8 Devido aos propósitos da pesquisa, não discutiremos sobre outras categorias de conceitos intuitivos abordados no $\mathrm{FCl}$. Caso o leitor queira aprofundar seus conhecimentos relativos aos assuntos tratados no $\mathrm{FCl}$ e aos distratores presentes nas questões, além de entender melhor a estrutura do inventário, ver Silval, Silva e Mansor (2009). 
apresentada na Figura 3. Dentre eles, podemos citar o ímpeto, que é o termo "utilizado pelos criadores do ICF para categorizar a idéia dos alunos que acham que há um tipo de 'força intrínseca' que mantém o movimento de uma partícula" (SILVAL; SILVA; MANSOR, 2009, p. 5).

Assim, utilizar a ideia de ímpeto para explicar o movimento de um corpo mostra que o estudante não consegue compreender bem a ideia da $1^{a}$ Lei de Newton. Segundo Mariconda e Vasconcelos (2006), essa dificuldade dos alunos em entender os conceitos newtonianos de movimento surge pelo fato de eles apresentarem, muitas vezes, concepções aristotélicas, o que torna complexo o entendimento das concepções de movimento propostas por Galileu.

Nesse ponto do estudo, julgamos conveniente fazer observações de ordem metodológica sobre a pesquisa, tarefa em que nos deteremos em nossa próxima seção.

\section{PROCEDIMENTOS METODOLÓGICOS}

Conforme afirmamos, essa pesquisa foi desenvolvida em uma escola particular de Fortaleza-CE. A instituição em foco tem turmas que vão da educação infantil até o Ensino Médio e funciona nos turnos matutino e vespertino. O professor que ficou encarregado de ministrar as aulas é licenciado em física pelo Instituto Federal de Educação, Ciência e Tecnologia do Ceará (IFCE).

Para o estudo, foram selecionadas duas turmas do $1^{\circ}$ ano do Ensino Médio, totalizando 44 sujeitos de pesquisa - dos quais 19 formaram o Grupo Experimental (GE) e 25, o Grupo Controle (GC) -, com faixa etária variável entre 14 e 16 anos de idade.

Como não foi possível utilizar a aleatoriedade na escolha dos alunos de cada uma das turmas - ou seja, as turmas já haviam sido formadas antes da intervenção do pesquisador -, utilizamos aqui um delineamento quaseexperimental, por este se mostrar mais adequado ao tipo de pesquisa que nos propusemos a fazer (CAMPBELL; STANLEY, 1979). Este delineamento é um 
dos mais usados na pesquisa educacional que envolve dois grupos a serem analisados (CAMPBELL; STANLEY, 1979; RIBEIRO; VERDEAUX, 2013). De acordo com Moreira (2011), neste tipo de delineamento, o pesquisador controla apenas a decisão sobre qual dos grupos deverá receber o tratamento (metodologia de ensino que se deseja avaliar) e qual dos grupos continuará recebendo aulas tradicionais, como comumente vem recebendo.

A Figura 4, exposta abaixo, mostra o modelo do delineamento quaseexperimental proposto por Campbell e Stanley (1979) e por Moreira (2011) envolvendo Grupo Controle (GC) e Grupo Experimental (GE):

FIGURA 4 - Modelo de delineamento quase-experimental

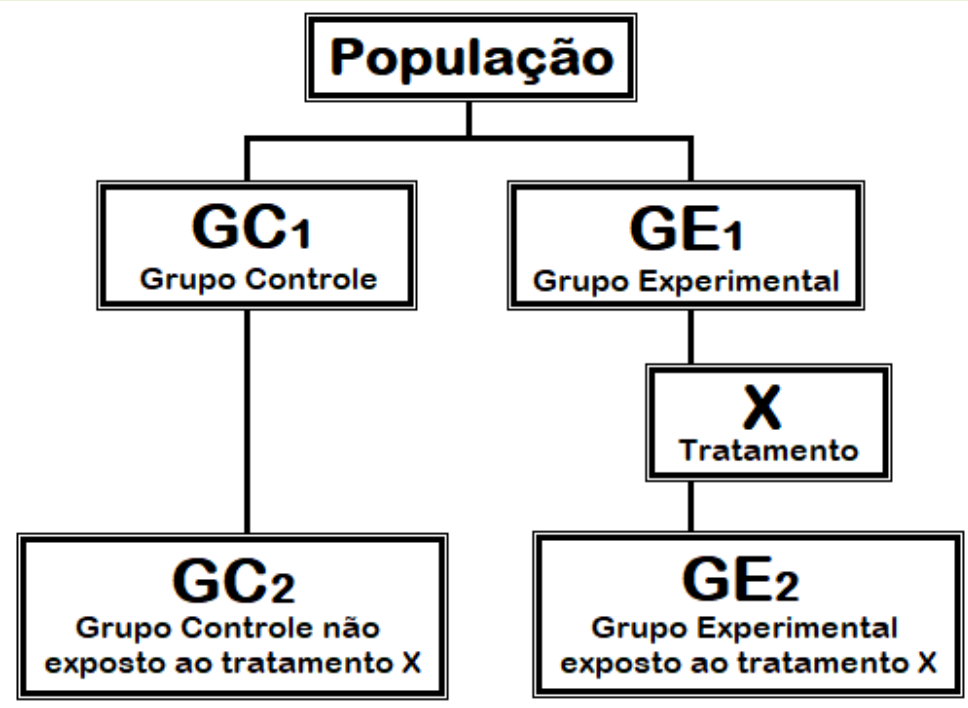

Fonte: Elaborado pelos autores, com base em Campbell e Stanley (1979) e por Moreira (2011).

Esse delineamento trabalha com dois grupos: um Grupo Experimental (GE) e um Grupo Controle (GC). É aplicado um pré-teste (1), antes da aplicação das metodologias, no Grupo de Controle 1 (GCl) e no Grupo Experimental 1 (GE1). De acordo com Campbell e Stanley (1979), a aplicação deste pré-teste é muito importante para o delineamento quaseexperimental, pois, como a escolha dos grupos não foi aleatória, os resultados desse teste podem evidenciar que os grupos estavam nas mesmas condições antes do tratamento. 
Após a aplicação do pré-teste, o GC é submetido às aulas tradicionais sobre o tema proposto na pesquisa, enquanto que o GE é submetido ao tratamento $X$ (metodologia de ensino que se deseja avaliar). Ambas as metodologias contemplarão um mesmo tema a ser ensinado. Ao final das aulas, aplica-se um pós-teste (2) nos dois grupos - Grupo de Controle e Grupo Experimental. Ainda a esse respeito, vale ressaltar que o pós-teste é o mesmo aplicado anteriormente - ou seja, é igual ao pré-teste - e que a diferença dos resultados entre o pré-teste e o pós-teste deverá fornecer, ao pesquisador, evidências sobre o efeito do tratamento X (MOREIRA, 2011).

Dessa forma, o plano de aula desta pesquisa seguiu o delineamento quase-experimental proposto por Campbell e Stanley (1979) e por Moreira (2011) e as aulas foram ministradas no período compreendido entre os dias 10 de setembro e 9 de outubro de 2018, totalizando 14 horas/aula para cada grupo, de acordo com o calendário anual já previsto para as aulas de Física nas turmas. Portanto, não foi preciso alterar o planejamento de ensino já formulado pelo professor no início do ano letivo.

O Grupo Controle (TC) teve 14 aulas expositivas tradicionais, em que o professor utilizou apenas o quadro branco e o pincel. Ao final de cada encontro, foi direcionada uma atividade para casa com exercícios do próprio livro didático adotado pelo colégio. O Grupo Experimental (TE) teve a mesma quantidade de aulas, mas estas foram divididas da seguinte forma:

- Sete aulas expositivas com a utilização, por parte do professor, das simulações PhET projetadas na lousa através de um DataShow. Ao final de cada encontro, foi direcionada uma atividade para casa com exercícios 9 propostos pela própria plataforma PhET.

- Sete aulas interativas no laboratório, com a utilização das simulações PhET por parte do professor e dos alunos, no laboratório de informática do colégio. Nestes encontros, foi disponibilizado um computador para cada um dos alunos, que, com a orientação do professor, simulou

\footnotetext{
9 O acesso às atividades do PhET é restrito para professores. Caso o professor queira acessar as atividades, precisará fazer o cadastro na página: https://phet.colorado.edu/en/teachingresources/browse-activities.
} 
situações hipotéticas de movimentos de corpos nas simulações Forces and Motion: Basics (HTML5) e Projectile Motion (HTML5). É importante ressaltar que, a cada assunto discutido no laboratório de informática, o professor estimulou o trabalho colaborativo, incentivando os estudantes, divididos em grupos de três, a pensar e a trocar ideias entre si e com o próprio professor. Ao final de cada aula, foi direcionada uma atividade para casa com exercícios propostos pela própria plataforma PhET.

Abaixo, na Figura 5, apresentamos uma imagem dos alunos explorando a simulação Forces and Motion: Basics (HTML5):

FIGURA 5 - Imagem dos alunos interagindo com a simulação Forces and Motion: Basics (HTML5)

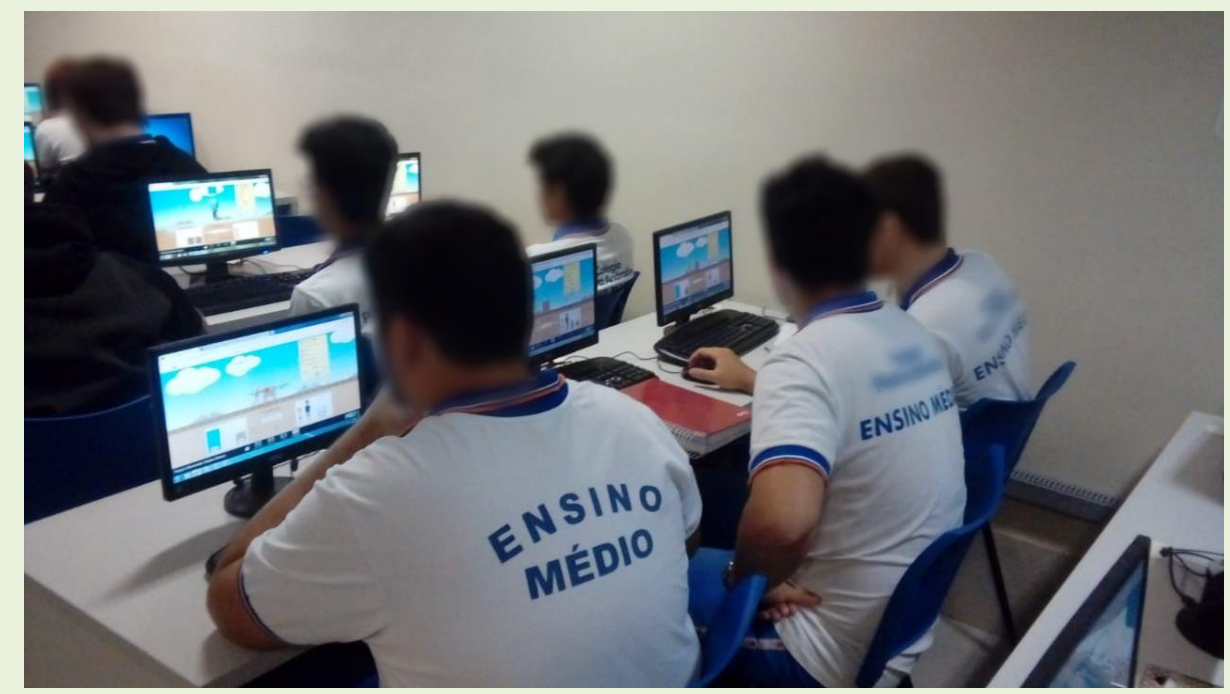

Fonte: Elaborado pelo autor.

É pertinente indicar, ainda, que as aulas tradicionais com a utilização, por parte do professor, das simulações PhET e as aulas interativas com a utilização, por parte do professor e dos alunos, das simulações PhET foram intercaladas umas com as outras.

Também vale ressaltar que o professor que lecionou as aulas nas duas turmas, durante esse período, não teve acesso ao $\mathrm{FCl}$, para que não fosse influenciado a adaptar as suas exposições lou a forma como guiaria as 
aulas) à resolução do teste e influenciasse, indiretamente, nas respostas dadas pelos estudantes por ocasião do pré-teste e do pós-teste.

Apresentados os aspectos teóricos e metodológicos da pesquisa nas seções anteriores, resta-nos, na próxima seção do estudo, realizar a análise dos dados coletados.

\section{ANÁLISE DOS DADOS}

Nesta seção, analisaremos os dados obtidos a partir do pré-teste e do pós-teste que foram aplicados, respectivamente, no primeiro e no último dia de aula e que, como já foi mencionado anteriormente, constituíram-se das mesmas questões do $\mathrm{FCl}$. Vale ressaltar que não foram utilizadas as 30 questões do $\mathrm{FCl}$, pois não tínhamos o propósito de explorar todos os assuntos abordados no teste completo.

Assim, os softwares escolhidos simulam apenas situações relacionadas a alguns conteúdos tratados no teste completo. Dessa forma, selecionamos 21 questões do $\mathrm{FCl}$ para formularmos os testes (pré e pós).

A Tabela 1, exibida abaixo, mostra a relação entre os conteúdos abordados no pré e no pós-teste e as questões que se referem a esses assuntos:

TABELA 1 - Relação entre os conteúdos abordados no pré e no pós-teste e as questões referentes a esses assuntos

\begin{tabular}{cc}
\hline Conteúdo & Questões do FCI \\
\hline $1^{a}$ Lei de Newton & $1,16,21,23,24$ \\
\hline $2^{a}$ Lei de Newton & $3,4,12,15,16,21,22,25,26$ \\
\hline $3^{a}$ Lei de Newton & $4,15,16,28,29$ \\
\hline Cinemática & $12,13,14,19,20,26,27$ \\
\hline Força Gravitacional & $1,2,3,12,13,14,29,30$ \\
\hline Força de Atrito & $1,2,3,25,27,30$ \\
\hline
\end{tabular}

Fonte: Elaborado pelos autores.

A Tabela 1 mostra que as 21 questões selecionadas para o pré-teste e para o pós-teste que foram aplicados na Turma Controle (TC) e na Turma Experimental (TE) englobam conceitos relacionados às Leis de Newton, à Cinemática e às forças gravitacionais e de atrito. Além disso, podemos 
observar que, para acertar algumas das questões, os alunos precisavam ter um conhecimento específico de mais de um conteúdo simultaneamente.

O Gráfico 1, apresentado abaixo, por sua vez, mostra o número de acertos da Turma Controle para cada uma das questões 10 dos testes aplicados. É importante ressaltar também que esta turma é formada por 25 alunos, mas, destes, só foram analisadas as respostas de 22 , número que corresponde à quantidade de estudantes que fizeram tanto o pré-teste quanto o pós-teste.

GRÁFICO 1 - Resultados obtidos após a aplicação do pré-teste e do pósteste na Turma Controle (TC)

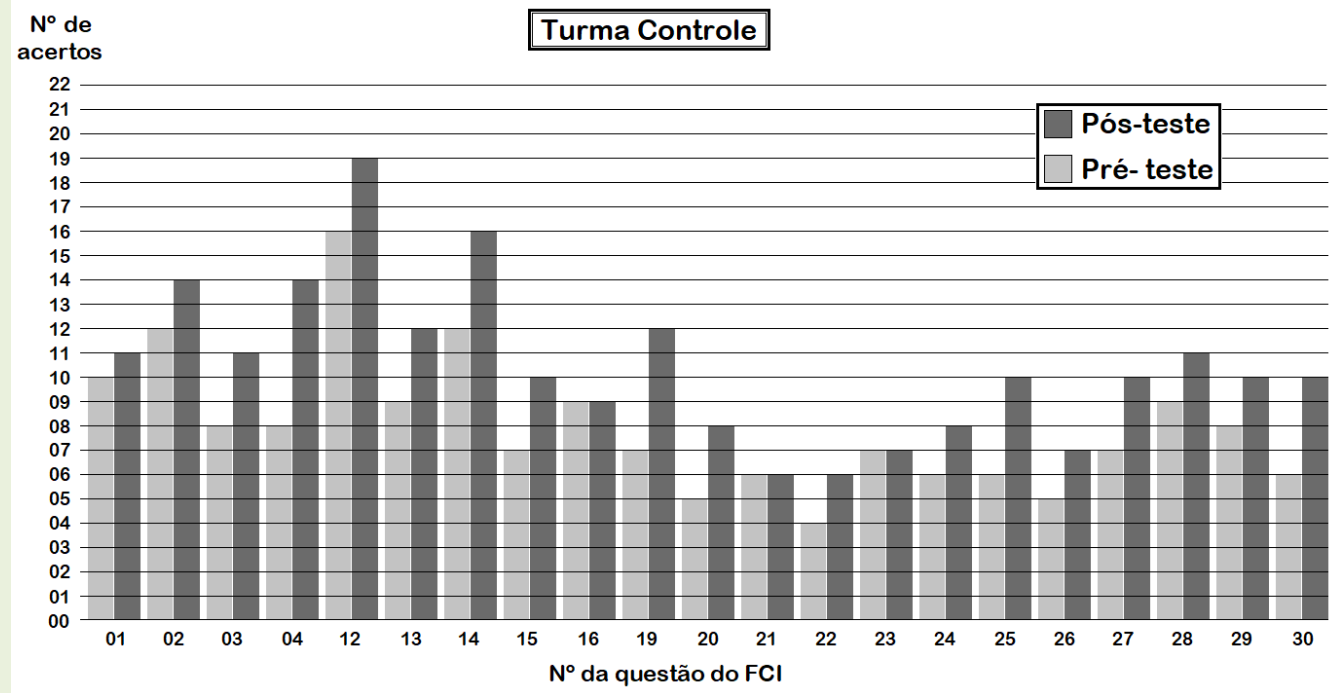

Fonte: Elaborado pelos autores.

Os resultados apresentados no Gráfico 1 mostram que, após as aulas tradicionais, houve uma melhor compreensão dos conceitos newtonianos por parte dos alunos da TC, visto que o percentual de acertos do pós-teste supera o do pré-teste para quase todas as questões. Por outro lado, o conceito de inércia, que está relacionado à primeira Lei de Newton, ainda pareceu ser algo abstrato para os alunos, visto que não houve um aumento percentual considerável no número de acerto das questões do teste que

\footnotetext{
10 No pré-teste e no pós-teste, as questões foram numeradas de 1 a 21. Entretanto, o Gráfico 1 mostra o número da questão conforme consta no $\mathrm{FCl}$, para que, caso o leitor tenha interesse, possa analisar, de forma mais específica, o seu conteúdo.
}

Revista Exitus, Santarém/PA, Vol. 10, p. 01-27, e020020, 2020. 
tratam deste conceito, quando comparamos o desempenho dos alunos no pré e no pós-teste.

O Gráfico 2, apresentado abaixo, mostra o número de acertos da Turma Experimental para cada uma das questões do pré-teste e do pós-teste aplicados. É importante afirmar que a Turma Experimental é formada por 19 alunos, mas, destes, só foram analisadas as respostas dos que fizeram tanto o pré-teste quanto o pós-teste, totalizando 17 alunos.

GRÁFICO 2 - Resultados obtidos após a aplicação do pré-teste e do pósteste na Turma Experimental

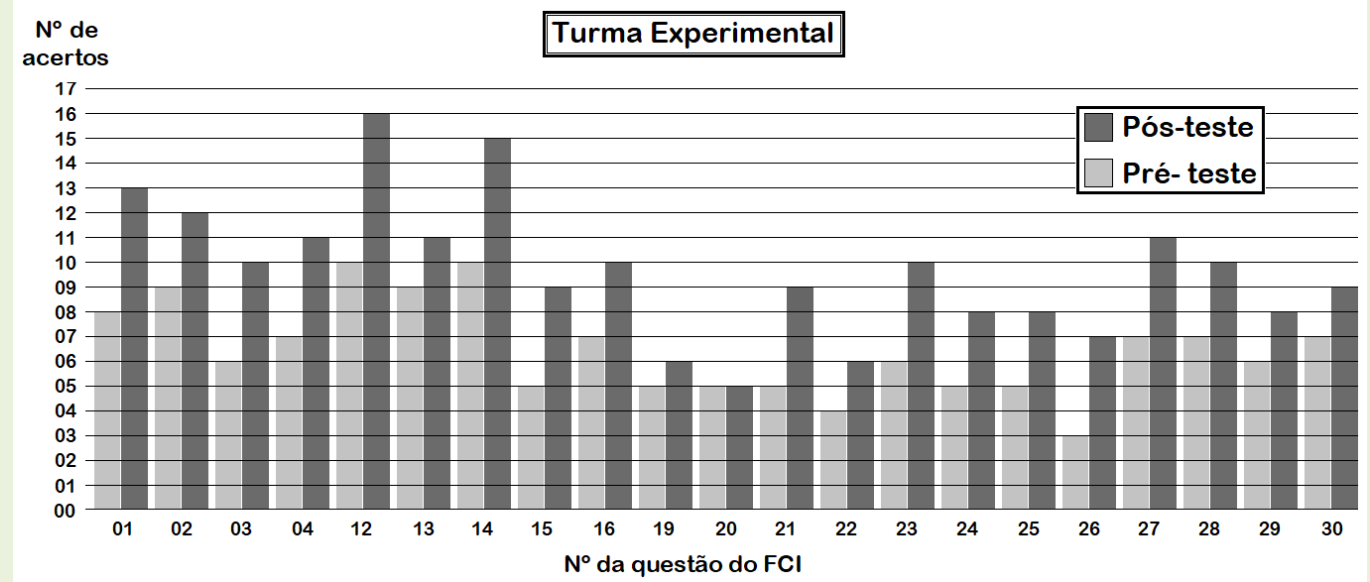

Fonte: Elaborado pelo autor.

Os resultados apresentados no Gráfico 2 mostram que, após as aulas na TE - nas quais os alunos puderam interagir com as simulações -, também houve uma melhor compreensão dos conceitos newtonianos, visto que o percentual de acertos do pós-teste supera o do pré-teste para quase todas as questões.

O percentual de acertos da TE relativo às questões que envolvem os conceitos de inércia e de movimento dos corpos, desprezando o atrito, foi bem superior ao da Turma Controle. Um dos fatores que podem ter influenciado para esse resultado é que as simulações conseguem criar situações de movimento que são difíceis de serem imaginadas, como é o caso dos movimentos sem atrito, por exemplo.

No ensino tradicional, os estudantes aprendem os conceitos físicos observando imagens estáticas e, muitas vezes, constroem modelos mentais 
incompletos ou incorretos, que dificultam uma aprendizagem mais profunda dessas noções (BEICHNER, 1997). Para os estudantes que não entendem bem os conceitos de inércia e da $1^{a}$ Lei de Newton, por exemplo, é difícil compreender que o fato de que um corpo se mantém em movimento não implica, necessariamente, na existência de uma força resultante diferente de zero agindo sobre ele.

Por outro lado, o desempenho da TE referente ao aumento percentual de acertos das questões 19 e 20, que envolvem conceitos de Movimento Retilíneo Uniforme (MRU) e Movimento Retilíneo Uniformemente Variado (MRUV), foi quase insignificante em comparação aos da TC. Ainda a esse respeito, convém indicar que, como os softwares escolhidos não apresentam simulações específicas sobre o MRU e o MRUV e como as aulas interativas baseavam-se nessas simulações, os alunos da TC - que tiveram aulas puramente expositivas - tiveram maior vantagem em relação aos da TE, em razão daqueles terem tido um período maior para trabalhar esses assuntos.

Ainda a esse respeito, é importante destacar outros trabalhos em que o uso das simulações PhET contribuíram de forma positiva no processo de ensino-aprendizagem das ciências da natureza. No campo da Física, Miranda (2013) desenvolveu aulas com seis simulações da plataforma PhET para ensinar Ondulatória. Os resultados deste trabalho mostraram que houve uma melhor compreensão dos conceitos pelos alunos que interagiram com as simulações durante as aulas em comparação com o desempenho daqueles que foram expostos às aulas tradicionais. Já no campo da Química, Mendes (2015) utilizou o software PhET como ferramenta para o ensino de Balanceamento de Reações Químicas e comparou o desempenho de duas turmas através de testes. Os resultados mostraram que "Como estratégia de ensino, o PhET revelou ser também eficiente da mesma forma que o uso de jogos lúdicos" (MENDES, 2015, p. 56).

Com nossa pesquisa, também pudemos observar que houve uma melhor compreensão dos conceitos por parte dos alunos da TE em comparação aos da TC, de maneira geral. Mas para comparar, de forma quantitativa, o rendimento e a evolução da TE com o rendimento e a 
evolução da TC, julgamos interessante utilizar o parâmetro denominado ganho normalizado (g). Este cálculo foi introduzido e validado por Hake (1998) para comparar o ganho de aprendizagem de turmas submetidas a diferentes tipos de metodologias e que, antes e depois desses processos metodológicos, foram avaliadas com um pré e com um pós-teste. A expressão matemática que define o ganho normalizado ou ganho de Hake (1998) é dada por:

$$
g=\frac{(\% p o ́ s)-(\% p r e ́)}{(100 \%)-(\% p r e ́)}
$$

Na expressão, "(\%pré)" e "(\%pós)" correspondem, respectivamente, ao percentual de acertos no pré e no pós-teste. Assim, o ganho normalizado (g) é a razão entre o ganho percentual real (diferença entre o percentual de acerto dos dois testes) e a máxima possibilidade de ganho médio. A Tabela 2, exibida abaixo, mostra os resultados dos cálculos do ganho normalizado para as duas turmas pesquisadas nesse trabalho:

TABELA 2 - Relação de sujeitos de pesquisa, porcentagem de acerto da TC e da TE no pré-teste e no pós-teste e ganho normalizado para ambas as turmas

\begin{tabular}{ccc}
\hline Turma & TC & TE \\
\hline No $^{\circ}$ de alunos que fizeram os dois testes (pré e pós) & 22 & 17 \\
\hline Porcentagem de acertos no pré-teste & 36,1 & 38,1 \\
\hline Porcentagem de acertos no pós-teste & 47,8 & 57,1 \\
\hline Ganho Normalizado & 18,3 & 30,7 \\
\hline
\end{tabular}

Fonte: Elaborado pelo autor.

Hake (1998) define três classes de ganho normalizado: baixo, médio e alto. Para ele, as turmas de ganho baixo apresentam valores de $g$ entre zero e trinta $(0<g<30)$; as turmas de ganho médio possuem valores de $g$ iguais ou maiores que trinta e menores que setenta $(30 \leq \mathrm{g}<70)$, e as turmas de ganho alto têm valores de $g$ iguais ou maiores que setenta $(70 \leq \mathrm{g})$. Se compararmos os valores de $\mathrm{g}$ obtidos nas turmas pesquisadas em nosso trabalho com os valores de $g$ propostos por Hake (1998), perceberemos que 
a Turma Controle teve um ganho baixo $(g=18,3)$ e que a Turma Experimental teve um ganho médio $(g=30,7)$.

Outros trabalhos também verificaram o ganho normalizado para turmas que foram submetidas a algum tipo de engajamento interativo, com base no cálculo proposto por Hake (1998). Savinainen e Scott (2002), por exemplo, obtiveram o valor de $g=0,57$ para turmas que foram submetidas às aulas com engajamento interativo, enquanto que Barros et al. (2004) encontraram valores de $g=0,19$ para turmas que foram submetidas às aulas tradicionais e $g=0,32$ para turmas com engajamento interativo. Podemos afirmar, em vista disso, que os resultados obtidos por Barros et al. (2004) para o ganho normalizado são bem semelhantes aos encontrados em nossa pesquisa.

Por fim, também consideramos interessante comentar o que foi percebido pelo professor durante as aulas na Turma Experimental. Por mais que, até então, a maioria dos alunos não conhecesse ou nunca tivesse acessado a plataforma PhET, eles se familiarizaram rapidamente com as simulações e fizeram muitas perguntas ao professor no decorrer das aulas, como, por exemplo, "Por que a força de atrito é alterada quando o bloco entra em movimento?" ou "Como é possivel movimentar uma geladeira com uma força tão pequena?". Muitos alunos mostraram-se animados em simular os mais diversos tipos de movimento nas duas simulações trabalhadas em sala de aula e ficaram curiosos em explorar outras simulações dentro da plataforma ao final das atividades.

\section{CONSIDERAÇÕES FINAIS}

O processo de ensino-aprendizagem sempre foi algo bastante complexo. No período atual, em que a tecnologia já é algo bem familiar para os jovens, é importante que o professor seja capaz de utilizá-la em sala de aula ao seu favor. As simulações computacionais educativas, em meio a diversos outros recursos pedagógicos, enquadram-se nas novas tecnologias. Além disso, são muitos os trabalhos que mostram que as simulações, como ferramenta de apoio no processo de ensino-aprendizagem, geram 
resultados positivos (HENNESSY et al., 1995; JIMOYIANNIS; KOLMIS, 2001; FINKELSTEIN, 2005; ARANTES, 2010; WIEMAN, 2014).

Os resultados obtidos nessa pesquisa são semelhantes àqueles apresentados nas pesquisas citadas. Nossos dados permitem a interpretação de que o uso das simulações computacionais da plataforma PhET, em associação com as aulas expositivas, trouxe benefícios para o processo de ensino-aprendizagem de Física (mais especificamente, para a mecânica newtoniana).

O uso dessa tecnologia virtual no ambiente da sala de aula promoveu o engajamento dos alunos nas atividades e uma aprendizagem mais ativa, pois eles trabalharam de forma colaborativa - entre si e com o professor -, explorando as simulações. Isso nos mostra o quão produtivo é o uso dessa ferramenta no ensino, sobretudo quando falamos do ensino de Física, que se marca pela existência de abstrações em quase todos os assuntos abordados, como, por exemplo, a força de atrito (mencionada anteriormente), alguns fenômenos que acontecem no ambiente microscópico ou até mesmo fenômenos invisíveis aos nossos olhos, como as ondas de rádio.

Vale destacar que não é apenas a utilização das simulações por parte dos alunos que fará com que o aprendizado seja eficaz. Deve-se levar em consideração que os alunos terão dificuldades e dúvidas referentes aos fenômenos que serão explorados no software. Assim, a presença e a intervenção do professor no momento da utilização das simulações é fundamental para a aprendizagem do aluno (SANTOS, 2017). Conforme consta nos próprios Parâmetros Curriculares Nacionais (PCN) (BRASIL, 2002, p. 229-230).

A escola não pode ficar alheia ao universo informatizado se quiser, de fato, integrar o estudante ao mundo que o circunda, permitindo que ele seja um indivíduo autônomo, dotado de competências flexíveis e apto a enfrentar as rápidas mudanças que a tecnologia vem impondo à contemporaneidade (BRASIL, 2002, p. 229-230).

Finalmente, torna-se importante afirmar que seria interessante a elaboração de novas investigações sobre a eficiência das simulações no 
processo de ensino-aprendizagem em turmas do Ensino Básico. Nesse sentido, amostras mais representativas combinadas com uma maior aleatoriedade na distribuição dos alunos nas turmas são fatores que influenciariam em uma pesquisa mais confiável e generalizável.

\section{REFERÊNCIAS}

ARANTES, A. R.; MIRANDA, M. S.; STUDART, N. Objetos de Aprendizagem no ensino de Física: usando simulações do PheT. Física na Escola, v. 11, n. 1, p. 27-31. 2010. Disponível em:

<http://www.sbfisica.org.br/fne/Vol1 1/Num1/a08.pdf> Acesso em: 10 de setembro de 2018.

ARTAMONOVA, I.; MOSQUERA-MOSQUERA, J. C.; MOSQUERA-ARTAMÓNOV, J. D. M. Aplicación de force concept inventory en América Latina para la evaluación de la comprensión de los conceptos básicos de mecánica a nivel universitário. Revista Educación en Ingeniería, v. 12, n. 23, p. 43-48. 2017. Disponível em:

<https://www.educacioneningenieria.org/index.php/edi/article/view/729/31 3>. Acesso em: 10 de setembro de 2018.

BARROS, J. A. et al. Engajamento Interativo no curso de Física da UFJF.

Revista Brasileira de Ensino de Física, v. 26, n. 1, p. 63-69. 2004.

BEICHNER, R. The impact of video motion analysis on kinematics graph interpretation skills. American Journal of Physics, v. 64, n. 10, p. 1272-1277. 1997.

BRANSFORD, J. D.; BROWN, A. L.; COCKING, R. R. How People Learn: Brain, Mind, Experience, and School. Washington, DC: National Academy Press, 2000.

BRASIL. Ministério da Educação, Secretaria de Educação Média e Tecnológica. Linguagens, códigos e suas tecnologias: orientações educacionais complementares aos parâmetros curriculares nacionais PCNS+. Brasília: 2002.

CABALLERO, M. D. et al. Comparing large lecture mechanics curricula using the Force Concept Inventory: A five thousand student study. American Journal of Physics, v. 80, n. 7, p. 638-644. 2012.

CAMPBELL, D. T.; STANLEY, J. C. Delineamentos experimentais e quaseexperimentais de pesquisa. Tradução de Renato Alberto T. Di Dio. São Paulo: EPU-EDUSP, 1979. 
CHARLES, E. S. et al. Les retombées de DALITE (Distributed Active Learning Interactive Technology Environment): un outil en ligne d'apprentissage par les pairs en physique au collegial. Disponível em: <https://dspacecdctest.inlibro.net/xmlui/bitstream/handle/1 1515/34789/CHARLES-E-etcoll_2015_Affiche.pdf?sequence=1\&isAllowed=y>. Acesso em: 10 de setembro de 2018.

CLARK, D. et al. Rethinking Science Learning Through Digital Games and Simulations: Genres, Examples, and Evidence. Disponível em: <https://www.academia.edu/493598/>. Acesso em: 10 de setembro de 2018.

FINKELSTEIN, N. D. et al. When learning about the real world is better done virtually: A study of substituting computer simulations for laboratory equipment. Physical Review Special Topic: Physics Education Research, v. 1, n. 1, p. 1-8, jul./dec. 2005.

HAKE, R. R. Interactive-engagement vs. traditional methods: A six-thousandstudent survey of mechanics test data for introductory physics courses.

American Journal of Physics, v. 66, n. 1, p. 64-74. 1998.

HALLOUN, I. A.; HESTENES, D. Common-sense concepts about motion.

American Journal of Physics, v. 53, n. 11, p. 1056-1065. 1985.

HELOU, R.; NEWTON, V. B.; GUALTER, J. B. Tópicos de Física, 2: termologia, ondulatória e ótica. Ed. 18. reform. e ampl. -- São Paulo: Saraiva, 2007.

HENNESSY, S. et al. A classroom intervention using a computer-augmented curriculum for mechanics. International Journal of Science Education, v.17, n. 2, p.189-206. 1995.

HESTENES, D. et al. Force Concept Inventory. The Physics Teacher, v. 30, n. 3, p. 141-158. 1992.

HESTENES, D.; HALLOUN, I. A. Interpreting the force concept inventory: A response to March 1995 critique by Huffman and Heller. The Physics Teacher, v. 33, n. 8, 502. 1995.

HOPKINSON, J.; NAKAHARA, H.; ABDULLA, R. Can weekly workshop physics labs change strongly held student misconceptions? Disponível em: <http://meetings.aps.org/Meeting/MAR18/Session/F20.5>. Acesso em: 23 de outubro de 2018.

JIMOYIANNIS, A.; KOMIS, V. Computer simulations in Physics teaching and learning: a case study on students' understanding of trajectory motion.

Computers \& Education, v. 36, n. 2, p.183-204. 2001. 
MAKUCH, F. B.; MARTINS, M. A. O uso do PhET Simulations no ensino de frações. Revista Brasileira de Ensino de Ciência e Tecnologia, v. 11, n. 2, p. 117, mai./ago. 2018.

MARICONDA P. R.; VASCONCELOS J. Galileu e a nova Física. São Paulo: Ed. Odysseus, 2006.

MEDEIROS, A.; MEDEIROS, F. C. Possibilidades e Limitações das Simulações Computacionais no Ensino de Física. Revista Brasileira de Ensino de Física, $v$. 24, n. 2, p. 77-86. 2002.

MENDES, A. P.; SANTANA, G. P.; PESSOA JÚNIOR, E. S. F. O uso do software PhET como ferramenta para o ensino de balanceamento de equação química. Revista Amazônica de Ensino de Ciências, v. 8, n. 16, p. 52-60, jan./jun. 2015.

MIRANDA, M. S. Objetos virtuais de aprendizagem aplicados ao ensino de Física: uma sequência didática desenvolvida e implementada nos conteúdos programáticos de Física ondulatória, em turmas regulares do nível médio de escolarização que utilizam um sistema apostilado. 2013. 126 f. Dissertação (Mestrado em Ensino de Ciências Exatas), Centro de Ciências Exatas, Universidade Federal de São Carlos, São Carlos, 2013.

MOREIRA, M. A. Metodologias de pesquisa em ensino. São Paulo/SP. Editora: Livraria da Física, 2011.

PAPALIA, D. A.; OLDS, S. W.; FELDMAN, R. D. Desenvolvimento humano. 12. ed. Porto Alegre: Artmed, 2013.

PERKINS et al. PhET: Interactive Simulations for Teaching and Learning Physics. The Physics Teacher, v. 44, n. 1, p. 18-23. 2006.

PODOLEFSKY, N. S.; PERKINS, K. K.; ADAMS, W. K. Computer simulations to classrooms: tools for change. Disponível em:

<https://www.compadre.org/per/items/1365.pdf>. Acesso em: 23 de outubro de 2018.

PRENSKY, M. C. Digital Natives, Digital Immigrants. Disponível em:

<http://www.marcprensky.com/writing/Prensky\%20-

\%20Digital\%20Natives,\%20Digital\%20lmmigrants\%20-\%20Part1.pdf>. Acesso em: 23 de outubro de 2018.

RUTTEN, N.; VAN JOOLINGEN, W. R.; VAN DER VEEN, J. T. The learning effects of computer simulations in science education. Computers \& Education, vol. 58, n. 1, p. 136-153. 2012.

RIBEIRO, J. L. P.; VERDEAUX, M. F. S. Uma investigação da influência da reconceitualização das atividades experimentais demonstrativas no ensino 
da óptica no ensino médio. Investigação em Ensino Ciências, v.18, n. 2, p. 239-262. 2013.

SANTOS, D. S. O uso de simulações no ensino de ciências: uma perspectiva para o ensino do efeito estufa. 22f. 2017. Trabalho de Conclusão de Curso (Especialização em Tecnologias da Informação e Comunicação Aplicadas à Educação), Centro de Artes e Letras, Universidade Federal de Santa Maria, Santa Maria, 2017.

SILVAL, T. H.; SILVA, G. S. F.; MANSOR, M. O uso do Inventário dos Conceitos de Força para análise das concepções de mecânica newtoniana de alunos de Licenciatura em Física. Disponível em: <

http://www.cienciamao.usp.br/dados/snef/_ousodoinventariodosconce.trab alho.pdf>. Acesso em: 23 de outubro de 2018.

STUDART, N. Simulação, games e gamificação no ensino de Física. Disponível em: <http://eventos.ufabc.edu.br/2ebef/wpcontent/uploads/2015/10/Studart_XXI_SNEF_Final_NEW.pdf>. Acesso em: 23 de outubro de 2018.

SALES, G. L. et al. Gamificação e ensinagem híbrida na sala de aula de Física: metodologias ativas aplicadas aos espaços de aprendizagem e na prática docente. Conexões: ciência e tecnologia, v. 11, n. 2, p. 45 - 52. 2017.

SAVINAIMEN, A.; SCOTT, P. Using the Force Concept Inventory to monitor student learning and plan teaching. Physics Education, v. 37, n. 1, p. 53-58. 2002.

WIEMAN, C. E.; PERKINS, K. K.; ADAMS, W. K. Oersted Medal Lecture 2007: Interactive simulations for teaching physics: What works, what doesn't, and why. American Journal of Physics, v. 76, n. 4-5, p. 393-399. 2008.

WIEMAN, C.; ADAMS, W. K.; PERKINS, K. K. PhET: Simulations that enhance learning. Disponível:

<https://phet.colorado.edu/publications/PhET_Simulations_That_Enhance_Le arning.pdf>. Acesso em: 23 de outubro de 2018.

WIEMAN, C. et al. Teaching Physics using PhET simulations. The Physics

Teacher, vol. 48, n. 4, p. 1-5. 2010.

WIEMAN, C. Large-scale comparison of science teaching methods sends clear message. Proceedings of the National Academy of Sciences (PNAS), v. 111 , n. 23, p. 8319-8320. 2014.

Recebido em: 19 de março de 2019

Aprovado em: 08 de agosto de 2019 